\title{
Selbstverwaltung der Gerichte: vertikal versus horizontal
}

\section{Historische Kontinuität eines hierarchischen Justizaufbaus}

Die der rechtsprechenden Gewalt in Deutschland vorgegebenen »institutionellen Strukturen « stammen aus dem vorletzten Jahrhundert. Aufbau und Funktionsweise der Gerichte in der Bundesrepublik werden noch heute - am Beginn des 21. Jahrhunderts - maßgeblich bestimmt durch das Gerichtsverfassungsgesetz (GVG) aus dem Jahre 1877. Damals schuf man »den Justizapparat« nach dem Bild des preußischen Obrigkeitsstaates: hierarchisch gegliedert und autoritär geführt. Die hierarchische Gliederung der Justiz entsprach der staatlichen und gesellschaftlichen Ordnung in der zweiten Hälfte des 19. Jahrhunderts. Sie überdauerte das Kaiserreich, spielte dann eine unrühmliche Rolle in der Weimarer Republik und erwies sich schließlich auch als ein geeignetes Strukturprinzip für den faschistischen Staat von 1933 bis 1945.

Aus der »Justizkatastrophe« (Adolf Arndt) im Dritten Reich wollte man beim Aufbau der Bundesrepublik verfassungsrechtliche Konsequenzen ziehen. Die »systembedingte Subalternität« des deutschen Richters, die ein wesentliches Element für das Versagen der Justiz war, sollte für immer beseitigt werden. Die Weimarer Verfassung hatte zwar die richterliche Unabhängigkeit in Artikel 102 garantiert, die Richter jedoch gleichzeitig den beamtenrechtlichen Bestimmungen unterworfen. Damit hatte man den »richterlichen Beamten « geschaffen, ein Richterbild, das aufgrund seiner inneren Widersprüchlichkeit scheitern musste.

Der Parlamentarische Rat wollte deshalb bei der Schaffung des Grundgesetzes den »kleinen Justizbeamten« der Weimarer Republik durch einen äußerlich und innerlich unabhängigen »neuen Richtertyp« ersetzen, um »den besonderen Charakter der Richter als der Repräsentanten der dritten staatlichen Gewalt deutlich herauszustellen «. Man wollte Richterpersönlichkeiten, die »ihr Amt im Geiste der Demokratie ausüben«, wie es zum Beispiel in der Hessischen Verfassung vorbildhaft heißt.

Doch die Herauslösung des Richters aus den hierarchischen Strukturen des Beamtentums ist nicht gelungen. Zwar wurden im Bund und in mehren Bundesländern parlamentarische Richterwahlausschüsse eingerichtet, die die demokratische Legitimation der Richterinnen und Richter stärkten. Das Gerichtsverfassungsgesetz aus dem Jahre 1877 wurde aber nicht entscheidend verändert und der Verfassungsauftrag des Art. 98 GG, die Rechtsstellung der Richter durch besonderes Gesetz zu regeln, wurde durch das Deutsche Richtergesetz von 1961 (DRiG) und durch die Richtergesetze der Länder nur unzureichend erfüllt. Es blieb bei der subsidiären Anwendung der beamtenrechtlichen Vorschriften, auch wenn in $§ 46 \mathrm{DRiG}$ schamvoll hinzugefügt ist, dass dies nur »bis zu einer besonderen Regelung « gelten soll, - eine Regelung die seit nunmehr einem halben Jahrhundert aussteht. Das Richteramtsrecht ist auch heute noch weitgehend nach beamtenrechtlichen Grundsätzen gestaltet. Es wurden lediglich einige Titel abgeschafft und eine eigene Besoldung für Richter eingeführt, doch es blieb bei dem beamtenähnlichen Laufbahn- und Beförderungssystem, das zu vielfältigen 
Abhängigkeiten führt. Das hatte zur fatalen Folge, dass auch unter der Herrschaft des Grundgesetzes ein für die Laufbahn von Beamten geschaffenes, von Weisungsgebundenheit und Wohlverhalten geprägtes Beurteilungssystem für die Richterinnen und Richter Geltung erlangte.

Damit fehlt es in Deutschland bis heute an einer ausreichenden Sicherung der richterlichen Unabhängigkeit gegenüber der sogenannten Richterpersonalpolitik durch die Justizverwaltung. Hier - bei der »richterlichen Personalsteuerung « - liegt aber die offene Flanke der richterlichen Unabhängigkeit. Theodor Eschenburg hat die sozialpsychologische Wirksamkeit dieses Systems schon vor vielen Jahren auf den Punkt gebracht: »Wer befördert, befiehlt!«. Dazu hat das Bundesverfassungsgericht schon Anfang der 50er Jahre - im Rahmen der sogenannten Status-Diskussion - erklärt, dass »die ressortmäßige Gebundenheit der Richter in der sogenannten Justizverwaltung schwere Gefahren für die richterliche Unabhängigkeit heraufbeschwört« (Jahrbuch des Öffentlichen Rechts, Neue Fassung, Band 6, Seite 204).

\section{Systembedingte Anpassungsmechanismen von Richterinnen und Richtern}

Die Personalsteuerung der Richter durch die Justizverwaltung steht nicht nur im unlösbaren Widerspruch zur richterlichen Unabhängigkeit, sondern sie geht zudem nahtund schamlos in Ämterpatronage über. Viele Richterinnen und Richter erliegen dem systembedingten Anpassungsreiz oder dem systembedingten Anpassungsdruck. Was liegt für die Karriereorientierten näher, als sich die inhaltlichen Positionen der bereits Aufgestiegenen zu eigen zu machen, um so das Wohlwollen derer zu erringen, die über ihr »berufliches Fortkommen« zu entscheiden haben? So entsteht vielfach eine Art von »Anbiederungshierarchie « mit dem sattsam bekannten »vorauseilenden« oder »hinterherhastenden« Gehorsam. Karrieristen sind für die Justizverwaltung am leichtesten zu steuern.

Der ehemalige nordrhein-westfälische Justizminister Behrens hat in einem Interview mit der Zeitschrift »Betrifft Justiz« erklärt, dass er »gelinde gesagt, erstaunt darüber ... (war), welche Obrigkeitsgläubigkeit ... (ihm) in dem Geschäftsbereich Justiz entgegengeschlagen ist «. Das habe er bisher an keiner anderen Stelle so erlebt. Es sei »frappierend, dass in einem öffentlichen Aufgabenbereich, in dem die richterliche Unabhängigkeit und das Denken und Entscheiden in Unabhängigkeit eine solch große Rolle spielen, ... die Denkstrukturen (so) sehr ausgerichtet sind auf den Blick nach oben ...« (BJ 1998, S. 254). Diese systembedingten Anpassungsmechanismen werden von den meisten Richterinnen und Richtern verleugnet oder verdrängt. Sie werden auch nicht als schmerzliche Deformation ihrer Persönlichkeit empfunden, sondern ihr angepasstes Verhalten wird zur «staatstragenden Gesinnung « hochstilisiert und als wertvolle Charaktereigenschaft erlebt, die auch nicht im Widerspruch zu »ihrer « richterlichen Unabhängigkeit steht. Die richterliche Unabhängigkeit ist jedoch kein Recht des Richters, sondern eine Pflicht gegenüber dem Bürger: Sie ist eine dem Richter auferlegte Verantwortung zu eigenständigem Denken und Handeln. Ein Richter kann sich den bequemen Luxus des Dienens und Gehorchens nicht leisten und er darf sich nicht der Macht und den Mächtigen »anpassen«. 
Dazu Heribert Prantl (BJ 1994, S. 376): »Die deutsche Politik hat die Justiz im Griff. Und dieser Griff funktioniert auch deswegen so gut, weil die Justiz ihn gern leugnet und sich schon gar nicht dagegen wehrt. Nicht nur hohe und höchste Justizämter werden nach Parteiproporz vergeben. Bei fast jeder Beförderungsstelle spielt Parteipolitik eine große Rolle«. Anspruch und Wirklichkeit der richterlichen Unabhängigkeit klaffen in der Realität weit auseinander. Da Richter auch nur Menschen sind, macht es wenig Sinn, über menschliche Schwächen zu lamentieren oder Appelle an die Charakterfestigkeit des Einzelnen zu richten. Man sollte keine personalistischen Erklärungen für Systemfehler suchen. Die geschichtliche Erfahrung hat gezeigt, dass hier strukturelle Sicherungen notwendig sind, um die Unabhängigkeit der rechtsprechenden Gewalt zu gewährleisten.

Dies gilt umso mehr, als die Judikative nicht nur durch die Personalsteuerung, sondern auch noch in vielfacher anderer Hinsicht von der Exekutive abhängig ist: von der Organisation und der Ausstattung der Gerichte über den Einsatz des nicht richterlichen Personals bis hin zum Ablauf des täglichen Geschäftsbetriebs, von der Dienstaufsicht über die Verwendung der sachlichen Mittel bis hin zur Zuweisung der gesamten finanziellen Mittel, da die rechtsprechende Gewalt noch nicht einmal selbst ihre Haushaltsmittel bei der Legislative beantragen kann. All das liegt in der Hand der Justizverwaltung. Gelegentlich wird selbst aus Kreisen der Obergerichtspräsidenten kritisch angemerkt, dass »die Dritte Gewalt als Beute der Exekutive« angesehen und behandelt wird (Peter Macke, ehem. Präsident des Brandenburgischen OLG) und dass der Weg zur Unabhängigkeit der Dritten Gewalt daher »nur über die Leiche des Justizministers« führt (Paulus van Husen, ehem. Präsident des OVG Münster).

Es ist ein unlösbarer Widerspruch, dass die rechtsprechende Gewalt in Deutschland unabhängigen Richtern anvertraut ist, die Verwaltung der Gerichte und die Richterpersonalpolitik jedoch der Exekutive und damit letztlich der Regierung.

\section{Das Präsidium als erster Schritt zu einer selbstverwalteten Justiz}

Nur ein kleiner Teil der Gerichtsverwaltung ist in Deutschland der richterlichen Selbstverwaltung überlassen: dem von den Richtern des jeweiligen Gerichts aus ihrer Mitte heraus gewählten Präsidium. Es verteilt die Rechtsprechungsaufgaben unter den Richtern (die Geschäftsverteilung) und bestimmt die Zusammensetzung der Spruchkörper. Das demokratisch gewählte Präsidium (und die ihm zugrunde liegende sog. Präsidialverfassung) ist ein erster Schritt auf dem Weg zur Selbstverwaltung der Gerichte, dem aber noch viele Schritte folgen müssen. Denn auch das Präsidium bedarf der weiteren Demokratisierung und Enthierarchisierung.

Nach dem GVG ist selbst heute noch der vom Justizminister ernannte Gerichtspräsident »kraft Amtes « und mit Stimmrecht Vorsitzender des Präsidiums. Das bedeutet, dass das einzige nicht gewählte und damit nicht demokratisch legitimierte Mitglied dieses Gremiums neben Sitz und Stimme auch noch die Leitungsfunktion innehat. In diesem Zusammenhang muss hervorgehoben werden, dass die Präsidenten der Gerichte nicht die von unten gewählte Spitze der Richterschaft eines Gerichts sind, sondern dass diese Präsidenten vom Justizminister eingesetzt werden und quasi »als 
Spitze der Exekutive« von oben in die Gerichte hineinragen und den Einfluss der Ministerialverwaltung dort hineintragen. Sie vermitteln vielfach den Eindruck, als seien sie Ministerialbeamte mit externem Dienstsitz in den Gerichten. Sie üben überwiegend Verwaltungstätigkeit aus. Ihre Rechtsprechungstätigkeit hat - wie z.B. bei den Obergerichtspräsidenten - lediglich eine Feigenblattfunktion, die verschleiert, dass diese Präsidenten kaum noch Richter sind, sondern nahezu vollständig weisungsgebundene Beamtentätigkeit ausüben.

Bisher fehlt es in den Gerichten an einer homogenen Organisationsstruktur. Der vertikalen und hierarchischen Steuerungsorganisation der Justizverwaltung, deren bürokratische Weisungshierarchie vom Justizministerium über die Präsidenten der Gerichte (als »nachgeordnete Behörden«) und über die Geschäftsleiter bis hin zu der untersten Ebene der Mitarbeiter führt, steht eine in Ansätzen vorhandene demokratische und horizontale Selbstverwaltung der Richter gegenüber: das Präsidium.

Neben der Geschäftsverteilung haben die Richter jedoch keinen Einfluss auf die Arbeitsorganisation des Gerichts, die viele Richter als eine ihnen vorgegebene oder sogar aufgezwungene Justizbürokratie empfinden.

\section{Stärkung richterlicher Selbstverantwortung}

Wer - wie die Justizverwaltung - nur »in Erledigungszahlen« denkt und die bürokratisch-technische Effizienz eines quantitativ und nicht qualitativ verstandenen Justizsystems in den Vordergrund stellt, verkennt die Gefahr, dass solche Systeme für die davon betroffenen Menschen ins Inhumane umzuschlagen pflegen. Zudem sind derartige Systeme auf eine »institutionelle Unverantwortlichkeit« der handelnden Individuen angelegt, d. h. viele Richter fühlen sich letztlich nur noch für die Erledigung und nicht mehr für das Ergebnis ihrer Arbeit verantwortlich und sind entsprechend wenig motiviert. Doch nicht unmotivierte Rechtsbürokraten und halbmotivierte Rechtstechnokraten, die ohne Engagement »Akten bearbeiten « und »Fälle erledigen«, sondern engagierte Richterpersönlichkeiten werden in einem demokratischen Rechtsstaat gebraucht: Richter mit Einfühlungsvermögen, mit sozialer Kompetenz, mit Zivilcourage, mit dem Mut zur Gerechtigkeit, der sich auch gegen die Mächtigen in Staat und Gesellschaft erweisen muss.

Deshalb ist die wichtigste Ressource der Justiz zu aktivieren: die Motivation und das Engagement der Mitarbeiter. Das kann nur durch die Übertragung von Eigenverantwortung geschehen. Die Justiz muss vom Kopf auf die Füße gestellt werden. Die bürokratische Verwaltung und Kontrolle von oben muss ersetzt werden durch die Verantwortung, Kreativität und Flexibilität von unten.

Wir können es uns nicht länger leisten, dass diejenigen, die sich in unserer Justiz für Neuerungen offen zeigen und sich mit Engagement und Kritik einbringen, von der Justizverwaltung als Unruhestifter und Störenfriede ins Abseits gestellt werden.

Die altertümlichen, schwerfälligen, ineffizienten und demotivierenden hierarchischen Organisationsstrukturen der herkömmlichen Justizverwaltung müssen grundlegend verändert werden. 


\section{Enthierarchisierung der Justiz}

Hierarchie ist - nach Max Weber - eine Strukturform von Herrschaft, die auf institutionalisierter Ungleichheit beruht. Der Begriff ist religiösen Ursprungs und bedeutete ursprünglich »geheiligte Ordnung «. Später bezeichnete er eine »Herrschaft, die ihre Legitimation in sich trägt«. Hierarchie legitimiert und stabilisiert damit ein bestehendes Herrschaftssystem unter Berufung auf nicht mehr hinterfragbare Vorgegebenheiten. Heute wird der Begriff meist zur allgemeinen Bezeichnung von Über- und Unterordnungsverhältnissen gebraucht und zunehmend kritisch gesehen. Der lange Zeit nicht in Frage gestellte hierarchische Aufbau der Bürokratie in der öffentlichen Verwaltung und in der Wirtschaft wird heute mehr und mehr in Zweifel gezogen. Neuere betriebssoziologische und betriebspsychologische Untersuchungen haben gezeigt, dass die hierarchischen Strukturen für wachsende Probleme in Behörden und Unternehmen verantwortlich sind: Ressortdenken, Kommunikationsbarrieren, Zeit- und Ortsferne, sachfremde Entscheidungen auf »höherer« Ebene, Konkurrenzkämpfe untereinander, Anpassungsschwierigkeiten bei neu auftauchenden Problemen, Desinteresse und Demotivation der Mitarbeiter.

Heute werden deshalb dezentrale Strukturen - bei denen Entscheidungen vor Ort flexibel, zeitnah und in eigener Verantwortung getroffen werden können - als wesentlich effektiver und effizienter eingeschätzt. Hierarchien sind das falsche Leitbild für eine funktionsgerechte und unabhängige Dritte Gewalt in einem demokratischen Rechtsstaat. Die hierarchischen Justizstrukturen, die dem Obrigkeitsstaat des vergangenen Jahrhunderts näherstehen als dem demokratischen Rechtsstaat des Grundgesetzes, müssen endlich durch demokratische Strukturen ersetzt werden. Entsprechende Selbstverwaltungsmodelle wurden vom Deutschen Richterbund 2007, von den in der Gewerkschaft ver.di zusammengeschlossenen Richterinnen und Richtern 2003 und von der Neuen Richtervereinigung (NRV) bereits 1991 vorgelegt.

Für die NRV ist es schon seit langem an der Zeit, das Beförderungswesen endlich abzuschaffen. Alle Richterämter sind dann gleichwertig und werden auch gleich besoldet. Die Aufgaben des Vorsitzenden können vom Berichterstatter oder vom lebensbzw. dienstältesten Richter wahrgenommen werden. Die Personalsteuerung der Richter durch Regierung und Justizverwaltung ist damit endlich unterbunden.

Ohne das Beförderungsamt des Vorsitzenden gibt es dann auf übergerichtlicher Ebene nur noch Funktionszuweisungen, z.B. zu den verschiedenen Instanzen und Gerichtszweigen. Diese Funktionszuweisungen werden von einem demokratisch gewählten Selbstverwaltungsorgan vorgenommen: dem Gerichtsbarkeitsrat (z.T. auch Justizrat, Justizverwaltungsrat oder oberster Richterrat genannt). Die Gerichtsbarkeitsräte ersetzen auf Landes- und Bundesebene die für die Verwaltung der Gerichte bisher zuständigen Justizministerien der Länder und des Bundes. Sie bestehen zu zwei Dritteln aus von der Richterschaft gewählten richterlichen Mitgliedern und zu einem Drittel aus vom Parlament gewählten Parlamentariern (oder sonstigen im Rechtsleben erfahrenen Persönlichkeiten), die für vier oder fünf Jahre gewählt werden. Die Gerichtsbarkeitsräte übernehmen die Verwaltung der personellen und sachlichen Ressourcen - einschließlich der Haushaltsmittel und ihrer Anforderung vom Parlament - sowie das Disziplinarrecht. 
Für die Einstellung der Richter sind die Gerichtsbarkeitsräte nicht zuständig. Sie bleibt den Richterwahlausschüssen vorbehalten, die - umgekehrt wie die Gerichtsbarkeitsräte - zu zwei Dritteln aus vom Parlament gewählten Mitgliedern und nur zu einem Drittel aus von der Richterschaft gewählten Mitgliedern bestehen.

Um das noch einmal hervorzuheben: Wer in die Justiz will, der muss durch den $\mathrm{zu}^{2} / 3$ aus Parlamentariern bestehenden Richterwahlausschuss, um die demokratische Legitimation der einzustellenden Richterinnen und Richter zu gewährleisten und jede Kooptation auszuschließen. Wer aber innerhalb der Justiz welche Funktion wahrnimmt, das bestimmt der zu 2/3 aus Richtern bestehende Gerichtsbarkeitsrat, damit sichergestellt ist, dass weder die Legislative noch die Exekutive maßgeblichen Einfluss auf das Innenleben der Justiz ausüben und dadurch die Unabhängigkeit der Dritten Gewalt gefährden.

So wichtig der Richterwahlausschuss und der Gerichtsbarkeitsrat auch sind, der Schwerpunkt der zukünftigen Selbstverwaltung liegt für die NRV jedoch in den Gerichten vor Ort. Hier muss das Fundament der demokratischen Strukturen geschaffen werden, ohne die das Haus der Selbstverwaltung nicht gebaut werden kann. Es gilt, die Richterinnen und Richter in alle Entscheidungsprozesse mit einzubeziehen, die das Gericht betreffen. Die Eigenverantwortung für »ihr« Gericht muss bei ihnen geweckt werden, was nur möglich ist, wenn ihnen insoweit auch tatsächlich Rechte und Pflichten übertragen werden. Bisher besteht in Richterkreisen vielfach Unkenntnis und Desinteresse an der Verwaltung des Gerichts, da der einzelne Richter weder Einsicht noch Einfluss hinsichtlich der Verwaltungsvorgänge hat. Es gibt Richter, deren Interesse für das Gericht am Akteneingangsbock beginnt und am Aktenausgangsbock endet und deren persönliches Engagement sich in ihrer Karriereplanung erschöpft. Dies muss grundlegend geändert werden, wenn wir lebensnahe und weltoffene Richter wollen, die über den Tellerrand ihres Dezernats hinausblicken und Verantwortung für ihr Gericht tragen.

Dazu wird die schon in Ansätzen vorhandene Selbstverwaltung durch das Präsidium umfassend ausgebaut. Das Präsidium regelt in Zukunft nicht nur die Geschäftsverteilung, sondern es wird zum kollektiven Leitungsorgan, das alle organisatorischen und personellen Entscheidungen zur Verwaltung des Gerichts trifft (Grundsatz der Allzuständigkeit des Präsidiums). Den Vorsitz im Präsidium führt der Präsident, der aber natürlich nicht mehr vom Justizminister ernannt und auch nicht vom Gerichtsbarkeitsrat bestimmt, sondern von den Richtern des Gerichts gewählt wird - und zwar auf Zeit. Nach Ablauf der vier- oder fünfjährigen Amtszeit übt er wieder ausschließlich das Richteramt aus, was sich für eventuelle Größenphantasien als heilbar erweisen dürfte. Eine spätere (einmalige) Wiederwahl ist zulässig.

Die Aufgabe der Präsidentin bzw. des Präsidenten besteht darin, die Beschlüsse des Präsidiums auszuführen, die Verwaltung zu leiten sowie das Gericht nach außen zu repräsentieren. Die eigentliche Leitungsfunktion liegt jedoch beim Präsidium, in dem der Präsident - wie alle anderen Mitglieder - nur eine Stimme hat.

Damit erfolgt die Bewältigung der Aufgaben vor Ort durch die eigene Sachkunde der Richter des Gerichts, ohne dass sich Privilegien oder hierarchische Strukturen herausbilden können. Vor allem aber werden die Richterinnen und Richter bei der Ver- 
waltung des Gerichts lernen, sich nicht - wie bisher - als passives Objekt der Justizverwaltung, sondern als aktives Subjekt der Selbstverwaltung zu begreifen. Das erhöht ihr Engagement, ihr Verantwortungsgefühl und ihre Identifikation mit dem Gericht, was auch unter dem Gesichtspunkt der heute allseits angestrebten »corporate identity « nur begrüßt werden kann.

Darüber hinaus gilt es ein eigenes Berufsethos zu entwickeln, das auf dem Richterbild des Grundgesetzes aufbaut und aus dem Bekenntnis unserer Verfassung zu Demokratie und Gewaltenteilung, zum Rechts- und Sozialstaatsprinzip hergeleitet ist.

\section{Schlussbetrachtung}

Das in Deutschland bis heute bestehende System der Verwaltung der Gerichte durch die Exekutive ist mit der Unabhängigkeit der Dritten Gewalt nicht vereinbar. Dieses System entspricht nicht den Anforderungen, die an einen demokratischen, dem Gewaltenteilungsprinzip verpflichteten Rechtsstaat im Europa des 21. Jahrhunderts zu stellen sind. Die Gerichte sind daher aus der Fremdverwaltung der Exekutive zu lösen und in die Selbstverwaltung zu überführen.

Eine in diesem Sinne erneuerte Dritte Gewalt fördert die Motivation, das Engagement und das Verantwortungsbewusstsein der Richterinnen und Richter und hilft zu verhindern, dass sie zu subalternen Bürokraten und uneinfühlsamen Rechtstechnokraten werden. Eine in diesem Sinne erneuerte rechtsprechende Gewalt ist die Grundlage dafür, dass die Richterinnen und Richter des Grundgesetzes ihr Amt - auch in Zeiten der Krise - unabhängig und im Geiste der Demokratie ausüben können. 